\title{
CORRESPONDENCE
}

Electronic laboratory reporting to general practitioners; is there ROM for improvement?

Electronic communications hardware, such as fax machines and modems, is becoming cheaper, more powerful, and easier to use. Complex systems, such as the Epinet Lablink Host System (Vidicode, Cheltenham), are already available to send laboratory reports to general practitioners' surgeries, but they are expensive for both parties and a network would have to be large to be viable. Alternatively, secure "electronic mailboxes" could now be used economically for temporary storage of medically scrutinised reports for GPs to interrogate only as required. This could be combined with a routine postal service.

To assess the attitudes and access of our laboratory's GP users to this equipment we carried out questionnaire surveys in 1988 and 1991. Prompted by recent publications ${ }^{12}$ we also included questions relevant to audit of our general laboratory communications, and we tested the existing postal service.

In June 1988 we posted a questionnaire to 40 practices in Cambridgeshire. We asked if they had a fax machine or whether they intended to acquire one in the near future, and explored their attitudes to electronic reporting. Twenty $(50 \%$ response rate) replies were received. No practice had a fax and only one thought they would acquire one, but only two $(10 \%)$ were against the idea of electronic communications in principle. Concens were expressed about costs to the practice, confidentiality, and the permanence of printing on fax paper.

In July 1991 we sent a questionnaire to 170 doctors in 50 practices in and around Cambridgeshire. This time we also asked for comments on the postal transmission of reports; the appropriateness of our telephone contacts; the value of telephoning our laboratory for results and advice; possession of personal computers and modems; and whether practices intended to acquire any of this equipment.

Fifty nine GPs replied (35\% response) from 42 practices $(84 \%)$. All questions were not answered on every questionnaire, and several practices returned jointly. Only three of $42(7 \%)$ were dissatisfied with the postal service, $25(60 \%)$ were satisfied, and 14 $(33 \%)$ thought it good or excellent. No adverse comments were received about the telephone service, with our contacts being thought satisfactory by 47 of $51(92 \%)$ and good or excellent by four ( $8 \%$ ), and contacts originated by GPs satisfactory by 33 of 50 $(66 \%)$ and good or excellent by $17(34 \%)$.

By mid-1992 about half the GPs expected to have access to a fax machine, nearly $80 \%$ to a PC, and about one third to a modem. Only three $(5 \%)$ were against any form of electronic reporting. GPs who did not have a PC tended to specify "hard copy" of all reports, whereas the more computer literate required hard copy only until an electronic system had been proved reliable. This group also gave most support for the ability to access a practice specific "electronic mailbox". Many worries were expressed about the security of any electronic system, but a number wondered if the post was really any safer.
In June 1991 we included pre-stamped, return-addressed postcards within report envelopes sent to 49 local practices. (Of 44 $(90 \%)$ cards returned, all had been received within 24 hours. We intend to repeat this survey regularly.

Clearly, electronic communications apparatus is proliferating in surgeries, and this resource could be tapped to improve the quality of pathology reporting. Our surveys provided many morale-boosting responses, ${ }^{2}$ and highlighted a number of minor communication problems that we have attempted to remedy. Electronic mailboxes (together with a routine postal service) may be the most effective option at the moment, provided that laboratory processors were buffered from multiple on-line users, and that access to medical advice was preserved. Any new system will need high and proven reliability to surpass existing links. To quote one Cambridgeshire GP: “. . . all this would need a major change to our practice ... but the electronic office cannot be delayed indefinitely!"

M FARRINGTON
TG WREGHIT
MAT COLES
Clinical Microbiology and Public Health Laboratory,
Addenbrooke's Hospital,
Hills Road,
Cambridge CB2 2QW

1 Pedler SJ, Bint AJ. Survey of users' attitude to their local microbiology laboratory. J Clin Pathol 1991;44:6-9.

2 White PMB, Williams H, Richards J. Survey of general practitioners' attitudes to
microbiology services. J Clin Pathol 1991; 44:614-5.

\section{Acanthamoeba keratitis}

The interesting case report by Anderson and his colleagues ${ }^{1}$ serves as a useful reminder to laboratory workers of the importance of bearing this unusual potential diagnosis in mind when investigating lesions of the eye, especially in wearers of soft contact lenses. This report raises some further points which may be helpful to those wishing to investigate such cases.

The authors are quite right in saying that acanthamoebas are relatively tolerant of osmolarity: some free-living amoebae (FLA), especially Acanthamoeba spp, can readily be grown on a variety of media including nonnutrient water agar with a bacterial lawn, in mammalian tissue cultures, and some in axenic culture in complex media. ${ }^{2-6}$ Species vary, however, in their ability to grow in different media and even depending on the bacterial species used (Klebsiellas may be preferable as a pabulum). The morphological form of trophozoites and cysts may, in some species, vary, depending on the growth conditions, especially salinity. Growth at different temperatures will also tend to select for different species or strains.

Samples may contain a variety of species, not all potentially pathogenic. FLAs are ubiquitous in the environment, and in addition to their occurrence as contaminants in tissue cultures, the cysts may be found in the air (as demonstrated by slit-sample culture) and in tap water, both of which may provide a potential source of contamination for cultures as well as lenses and lens fluids. ${ }^{4578}$ The isolation of FLAs from such samples as lens fluid does not necessarily imply an aetiological role. Laboratory cultivation, therefore, needs to be performed, and interpreted, with great caution: definitive identification is a specialised task.

In addition to their role in the natural history of legionellosis, ${ }^{9}$ FLAs include species potentially capable of causing serious central nervous system infection. ${ }^{3}$ They should, therefore, be handled according to the recommendations of the Advisory Committee on Dangerous Pathogens. ${ }^{6}$

Species of FLA differ in their sensitivity to a variety of potential therapeutic compounds: results of tests by different authors have sometimes been conflicting and clinical response has often been disappointing. Propamidine (not Propanidine) isethionate, and hydroxystilbamidine isethionate have previously been shown to be effective against strains of Acanthamoeba as trophozoites, but cysts are considerably more resistant. ${ }^{25}$ In vitro studies on isolates, in a specialised laboratory, might be indicated to confirm identity, but drug susceptibility tests are not generally available. The Amoebiasis Unit will, on request, post medium to microbiologists wishing to attempt isolation, and will identify isolates and examine histological material.

The potential for environmental contamination also, of course underlines the need for great care in handling contact lenses and lens solutions, by those wearing them.

DP CASEMORE

Public Health Laboratory Glan Clwyd Hospital, Bodelwyddan, Clwyd LL195UJ DC WARHURST Amoebiasis Unit, Hospital for Tropical Diseases,
London NW1 OPE

1 Anderson D, Soo SS, Towler H. Acanthamoeba keratitis: experience in a non-specialist keratitis: experience in a non-sp
laboratory. J Clin Pathol 1991; $44: 699$.

2 Casemore DP. Sensitivity of Hartmanella (Acanthamoeba) to 5-fluorocytosine, hydroxystilbamidine, and other substances. J Clin Pathol 1970;23:649-52.

3 Warhurst DC. Pathogenic free-living amoebae. Parasitol Today 1985;1:24-8.

4 Warhurst DC, Mann PG. Acanthamoeba keratitis. Br Med J 1988;296:568

5 Kilvington S, Larkin DFP, White DG, Beeching JR. Laboratory investigation of Acanthamoeba keratitis. $J$ Clin Microbio 1990;28:2722-5.

6 Anonymous. Isolation and identification of Giardia cysts, Cryptosporidium oocysts and free living amoebae in water. London: HMSO 1989 \& 1990 .

7 Casemore DP. Free-living amoebae in home dialysis unit. Lancet 1977;ii:1078.

8 Stehr-Green JK, Bailey TM, Brandt FH, Carr $\mathrm{JH}$, Bond WW, Visvesvara GS. Acanthamoeba keratitis in soft contact lens wearers JAMA 1987;258:57-60.

9 Rowbotham TJ. Isolation of Legionella pneumophila from clinical specimens via amoebae, and the interaction of those and other isolates with amoebae. J Clin Pathol 1983;36:978-86.

\section{Urease activity of Helicobacter pylori}

Concerning the recently published paper in the Journal,' several authors have previously used acetohydroxamic acid as a specific urease inhibitor. Indeed, hydroxamic acid was established as a potent and specific urease inhibitor 30 years ago in our paper. ${ }^{2}$ Subsequently many other papers on the inhibitory effect of hydroxamic acid on urease activity of various origins and on the therapeutic application of hydroxamic acids have been published. The authors of the 
paper in your Journal did not refer to our original work but cite a newly published paper which is only a double-blind study of acetohydroxamic acid and does not provide any evidence that acetohydroxamic acid is a specific urease inhibitor. ${ }^{3}$ Anyone can easily search the literature using the keywords hydroxamic acid and urease inhibitor, or my name, to find lots of our papers.

I expect Editorial Boards to check cited references carefully. I am sending the same letter to the Editor of Gut. Would you please answer my protest.

K KOBASHI

Toyama Medical and Pharmaceutical University 2630 Sugitani Toyama-shi

Toyama 930-01 Japan

1 Goldie J, van Zanten SJO, Jalali S, Richardson $H$, Hunt RH. Inhibition of urease activity but not growth of Helicobacter pylori by acetolhydroxamic acid. J Clin Pathol 1991;44: $695-7$

2 Kyoichi Kobashi, Juni'ichi Hase, Kihachiro. Specific inhibition of urease by hydroxamic Specifo inhibilion of urease

willis. Biochim Biophys Acta 1962,65:380-3.

Williams John J, Rodman John S, Peterson Charles $M$. A randomized double-blind study of acetohydroxamic acid in struvite nephrolithiasis. N Eng J Med 1984;311:760-4.

Drs Veldhuyzen van Zanten and Goldie comment:

We are glad to respond to the concerns that are raised by Professor Kobashi. Maddox probably was right in saying "I suspect that a large part of the formal scientific literature is hardly every read at all" (', quoted from ${ }^{2}$ ). We are also a bit frustrated about the use of acetohydroxamic acid for the treatment of Helicobacter pylori, because we think we were among the first ones to consider it for this purpose. ${ }^{3}$ Apart from the feeling of lack of recognition, we blame ourselves for taking too much time in getting our data published.

"Parsimony with words is never a vice and rarely a fault". 5 Is this true for references to previous published work? Given the exponential growth of the scientific literature that is overwhelming the medical profession, editors of journals are increasingly trying to have published papers say more with less words. It is a good custom to only use relevant references which have been personally read by the author.

When our paper was accepted for publication the editors felt it could be shortened and fitted into the short reports section of the Journal. One of the requirements of short reports is to limit the number of references to 10. We are a little surprised that Professor Kobashi is dissatisfied since his work (although not the 1962 paper that he refers to survived the cut from the original number of 23 references to $13 .^{6}$ It is reference 6 in our paper and precisely refers to Professor Kobashi's work in stating that the inhibitory effect of acetohydroxamic acid is highly specific for urease. The first reference in his 1975 paper is the 1962 publication which Kobashi felt we should have used. Although we did see that reference, we never actually read it and therefore it was not used. With regard to the number of references, we in fact argued with the editors that a further reduction in the number of references to 10 would result in a loss of essential information and they allowed us to use 13 references. Finally, we did do computer searches on Medline, but unfortunately this database goes back only to 1966 and therefore we were unable to retrieve the 1962 reference this way.

It remains the obligation of authors to ensure that relevant literature is referenced in a fair and representative way, but we feel that restrictions on numbers of references sometimes could be an unwarranted constraint. Nevertheless, we do not think we gave Professor Kobashi reason to be disappointed but hope for him that this correspondence will mean a revival for his previous paper. Back to the future!

Maddox J. Tape or type? Lancet 1968;ii:1071.

Lock S. Thorne's better medical writing. 2nd edn. London: Pitman Medical Publishing Co Ltd, 1977:ix.

3 Goldie J, Jalali S, Velduyzen van Zanten SJO, Pickersgill J, Hunt RH. Effect of the urease inhibitor acetohydroxamic acid (AHA) on urease of Campylobacter pylori. Gastroenterology 1989;96:abstract 174.

4 Goldie J, Velduyzen van Zanten SJO, Jalali S, Richardson $\mathrm{H}$, Hunt $\mathrm{RH}$. Inhibition of urease activity but not growth of Helicobacter pylori by acetohydroxamic acid. $J$ Clin Pathol 1991;44:695-7.

5 Calnan J, Barabas A. Writing medical papers. A practical Guide. London: Heinemann Medical

6 Kobashi K, Takebe S, Terashima N, Hase J. Inhibition of urease activity by hydroxamic acid derivates of amino acids. J Biochem 1975;77:837-43.

\section{BOOK REVIEW}

Practical Statistics for Medical Research. DG Altman. (Pp 611; £32.) Chapman \& Hall. 1990. ISBN 0412276305.

Whereas statisticians see themselves as crusaders for scientific truth, research scientists, and particularly clinical trials organisers, see them occasionally going beyond the helpful and becoming lay preachers for unattainable perfection. The gap is largely the product of a language barrier and each party failing to understand the other's problems. The statistical referee who says that the paper would be acceptable only if the authors include another 2000 cases of Rare's disease with income-matched controls is being just as ridiculous as the scientist who trawls desperately to find a $p$ value of $<0.05$ somewhere in a mass of badly assembled data to give his paper editor appeal.

Doug Altman's book provides one of the best attempts so far at bridging the gap. Although aimed primarily at clinicians and scientists wanting to know more about statistics, the text also offers useful information to statisticians on the realistic limitations to be found in clinical research. There is much helpful background explanation, but where the book scores is that it uses real examples of real data, both in explaining how not to do it and in the carefully worked examples of the various techniques. There is useful advice on the use of microcomputers - the advantages and pitfalls-and, for those who really want to learn systematically, practical exercises at the end of each chapter. The answers are given at the back. Most of the tests commonly used in clinical research are well covered, and there are basic statistical tables provided for those who prefer to do their analyses by hand. Some, such as Cox's proportional hazards regression analysis, are coyly referred to as "more complex" than other sections of the book and it is made clear that such analyses require computer facilities. Finally there is a searing critique of the medical literature where common statistical errors are catalogued.

In short, it is the best book on statistics for medical research I have come across, in terms of both completeness and intelligibility. With one bound it takes the tyro to competence and enables the most uncritical reader to spot the "eight out of ten cat owners" statistics which are still depressingly prevalent in manuscripts submitted to medical journals.

JS LILLEYMAN

\section{NOTICES}

\section{ACP Locum Bureau}

The Association of Clinical Pathologists runs a locum bureau for consultant pathologists.

Applicants with the MRCPath who would like to do locums and anyone requiring a locum should contact The General Secretary, 221 Preston Road, Brighton BN1 6SA. Tel (0273) 561188. Fax: (0273) 541227

4th International Meeting on Trace Elements in Medicine and Biology

Trace Elements and Free Radicals in Oxidative Diseases

Chamonix, France

April 5-9, 1993

Main topics: Relations between trace elements and free radicals

Trace elements; zinc, selenium, iron, copper, free radicals, peroxidation, DNA, lipid, cancer, inflammation, aging, atherosclerosis, immunity ...

Official language: English

Simultaneous translation languages: French - English

Organised by: Society for free radical research (SFRR)

and Société Francophon d'Etude et de Recherche sur les Elements Trace Essentiels (SFERETE)

Local organiser: Club d'Etude sur les Radicaux Libres (CERLIB)

Registration information

Professeur Alain FAVIER Madame Arlette ALCARAZ Laboratoire de Biochimie C Hôpital A. MICHALLON B.P. $217 \mathrm{X}$ 38043 GRENOBLE Cédex 09 FRANCE

Tel: (33) 76765407

Fax: (33) 76426644 